\title{
Optimisation of aiming strategies in Solar Power Tower plants
}

\author{
Thomas Ashley ${ }^{a}$, , Emilio Carrizosa ${ }^{a}$, Enrique Fernández-Cara ${ }^{\text {b }}$ \\ a IMUS - Instituto de Matemáticas de la Universidad de Sevilla, Spain \\ ${ }^{\mathrm{b}}$ Dep. EDAN e IMUS, Universidad de Sevilla, Spain
}

\section{A R T I C L E I N F O}

\section{Article history:}

Received 1 March 2017

Received in revised form 20 June 2017

Accepted 28 June 2017

Available online 30 June 2017

\section{Keywords:}

Solar thermal power

Aiming strategy

Integer programming

\begin{abstract}
A B S T R A C T
The distribution of temperature on a Solar Power Tower (SPT) plant receiver directly affects the lifespan of the structure and energy generated by the plant. Temperature peaks and uneven distributions can be caused by the aiming strategy enforced on the heliostat field.

A non-optimised aiming strategy can lead to suboptimal energy generation and, more importantly, to risk of permanent damage to receiver components from thermal overloading due to sharp flux gradients.

In order to reduce damage to receivers and optimise the energy generation, an aiming strategy is developed which homogenises the flux distribution on a flat plate receiver in a SPT plant.

Results of a near real-time optimised aiming strategy are presented, demonstrating applicability to SPT plants of any size and shape, whilst also considering inclement weather conditions.
\end{abstract}

() 2017 Elsevier Ltd. All rights reserved.

\section{Introduction}

Research into renewable energy sources has continued to increase in recent years, and in particular the research and application of solar energy systems. Concentrated Solar Power (CSP) is a method of solar energy collection, where the energy from the Sun is concentrated by a field of heliostats onto a central receiver. In Solar Power Tower (SPT) plants, this receiver is mounted atop a tower, and the resultant thermal load is used to drive a steam generator. This allows high temperatures to be achieved and is an increasingly investigated method into renewable energy production, see Refs. [7,10,23,25].

The SPT plant is usually formed of at least one central tower, with a field of heliostats located in front of the receiver side of the tower. These heliostats are able to rotate, in order to track the movement of the Sun and focus the light onto the receiver surface. The chosen aiming point for the heliostats on the receiver surface will have an effect on the production of energy, as well as an effect on the lifetime of the materials used in the receiver surface, due to thermal stresses. Therefore, the aiming strategy used by an SPT plant is of importance when seeking to achieve the optimal energy

\footnotetext{
* Corresponding author. Instituto de Matemáticas de la Universidad de Sevilla (IMUS), Edificio Celestino Mutis - 1 Planta, Avda. Reina Mercedes, s/n, 41012 Sevilla, Spain.

E-mail addresses: tashley@us.es (T. Ashley), ecarrizosa@us.es (E. Carrizosa), cara@us.es (E. Fernández-Cara).
}

production, whilst minimising risk of damage to components.

The aiming strategy commonly used in research into the optimisation of SPTs assumes that all heliostats in the field aim at the centre of the receiver, see Ref. [11], a summary of various recent SPT optimisation techniques can be found in Ref. [7]. This assumption allows for easier computation of the flux distribution across the receiver surface and reduces complexity of the adjustment of the heliostats.

Using a central aim point for all heliostats leads to a large heat flux at the centre of the receiver and large flux gradients towards the edge of the receiver, which can cause strong heat loads and can lead to damage over time and therefore costly repairs [19-22,26].

An uneven flux distribution across the receiver surface also lowers the efficiency of the energy transfer to the thermal fluid within the receiver $[17,26]$. Therefore, maintaining an even distribution will increase the efficiency and allow for greater energy production.

Some research has been conducted where more complex aiming strategies are considered for different receiver types [5,8], as well as closed-loop feedback mechanisms to provoke changes in aiming strategy $[9,18]$. Applications of alternate optimisation algorithms for the aiming strategy have also been exhibited $[16,21,22,26]$ and a summary of optimisation techniques collected [6].

The distribution of reflected energy from the heliostats onto the receiver is assumed to be a Gaussian, as in Refs. [11,23]. The distribution can be written in the form: 
$f_{1}(x, y, \Theta) \exp \left(\frac{-f_{2}(u, v, x, y)}{2 f_{3}^{2}(t, x, y, \Theta)}\right)$

where the $f_{i}$ with $i=1,2,3$ are as follows:

$f_{1}(x, y, \Theta)=\frac{f_{4}}{2 \pi f_{3}^{2}(t, x, y, \Theta)\|\vec{w}\|^{2}}$

$f_{2}(u, v, x, y)=\frac{u^{2}+v^{2}}{2\|\vec{w}\|^{2}}\left[\left(1+f_{4}^{2}\right)+\frac{|u|}{\sqrt{u^{2}+v^{2}}}\left(1-f_{4}^{2}\right)\right]$

$f_{3}(t, x, y, \Theta)=\left[\mu_{1}^{2}+\left(\frac{\mu_{2}\left(1-f_{\cos }\right)}{4\|\vec{w}\|}\right)^{2}\right]^{1 / 2}$

and, finally,

$f_{4}(x, y)=\left\{\begin{array}{cc}0, & \text { if } \cos \beta \leq 0 \\ \frac{\vec{w} \cdot \vec{p}}{\|\vec{w}\|}, & \text { otherwise }\end{array}\right.$

In these definitions, it is assumed that the heliostat is at $(x, y)$ and aims at $(u, v)$, and $\vec{w}$ is the vector from the heliostat to the receiver. The cosine efficiency, $f_{\text {cos }}$, is given by:

$f_{\cos }(t, x, y, \Theta)=\sqrt{\frac{1}{2}+\frac{\vec{w} \cdot \vec{v}}{2\|\vec{w}\|}}$

where $\vec{v}$ is the solar vector with $\|\vec{v}\|=1$.

The cosine efficiency is a number between 0 and $1 ; f_{4}$ is equal to $\cos \beta$, where $\beta$ is the angle between the vector from heliostat to receiver and the vector normal to the receiver.

The fraction of reflected energy reaching the receiver, known as the spillage efficiency, can be found by integrating this distribution across the area of the receiver surface.

$f_{s p}(x, y, \Theta)=f_{1}(x, y, \Theta) \iint_{S} \exp \left(\frac{-f_{2}(u, v, x, y)}{2 f_{3}^{2}(x, y, \Theta)}\right) d u d v$,

where $S$ denotes the aperture area.

Converting this to polar coordinates with $u=\rho \cos \phi$ and $v=\rho \sin \phi$, gives:

$f_{s p}(x, y, \Theta)=f_{1}(x, y, \Theta) \int_{0}^{2 \pi} \int_{0}^{r} \exp \left(\frac{-\tilde{f}_{2}(\rho, \phi, x, y)}{2 f_{3}^{2}(x, y, \Theta)}\right) \rho d \rho d \phi$,

where $r$ is the radius of the circular receiver and $\tilde{f}_{2}(\rho, \phi, x, y) \equiv f_{2}(\rho \cos \phi, \rho \sin \phi, x, y)$.

The spillage efficiency, $f_{s p}(x, y, \Theta)$, is thus found by means of an exact integral over $\rho$ and then a numerical approximation over $\phi$; see Refs. $[14,15]$ for more information.

In order to reduce damage to receiver components and to optimise the energy reaching the receiver, multiple aim points across the receiver surface in a grid can be considered, as depicted in Fig. 1: a set of possible points within the receiver is given, and the solver must choose, for each heliostat and time instant, the most appropriate aiming point.

The field of heliostats would then be split, so that a proportion of them aim at each point. The result of this would be to smooth the flux gradient across the receiver surface and to reduce the peak

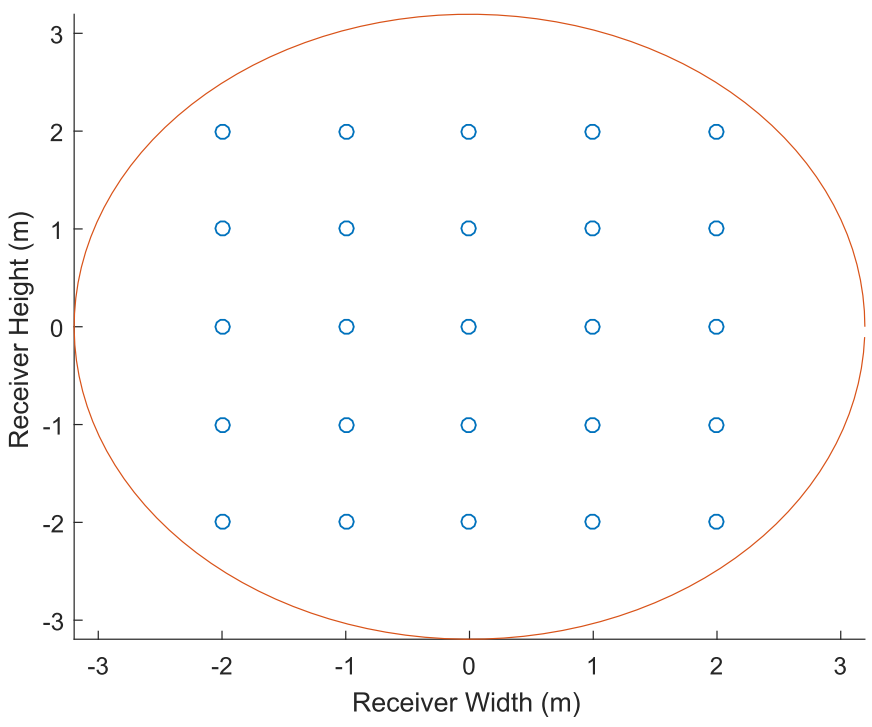

Fig. 1. Aim point grid.

heat flux at the centre.

Another effect of this strategy would be to increase the amount of energy that is being lost due to spillage, where aiming towards the edge of the receiver causes some of the energy to miss completely.

The goal of this study is to design an aiming strategy that minimises the flux gradient across the surface of the receiver, whilst minimising the spillage and maintaining a minimum amount of energy.

\section{Changing aim point}

In order to model the distribution of energy across the receiver surface when we consider an aiming point offset from the centre, the bounds of integration in (8) need to be altered, as shown below.

Consider the Gaussian distribution of the reflected energy as centered at the origin, and that the receiver is offset by aim point offset:

$f_{s p}(t, x, y, \Theta)=f_{1}(t, x, y) \int_{0}^{2 \pi} \int_{0}^{\widehat{r}(\phi)} \exp \left(\frac{-\tilde{f}_{2}(\rho, \phi, x, y)}{2 f_{3}^{2}(t, x, y, \Theta)}\right) \rho d \rho d \phi$,

where $\widehat{r}(\phi)$ is given by the solution of the equation $R^{2}=\widehat{r}^{2}+r_{0}^{2}-2 \widehat{r} r_{0} \cos (\theta-\phi)$.

We shall assume that the heliostat is always pointing within the receiver boundaries, if it is to be included in calculations (i.e. unless it is turned away for safety or other reasons and is then not producing output).

The spillage efficiency in (9) then becomes:

$$
\begin{gathered}
f_{s p}(t, x, y, \Theta) \approx \frac{2 \pi}{10} f_{1}(t, x, y) f_{3}^{2}(t, x, y, \Theta) \sum_{i=1}^{10} \frac{1}{f_{5}\left(\phi_{i}, x, y\right)}(1-\exp \\
\left.\left(-\frac{\widehat{r}\left(\phi_{i}\right)^{2} f_{5}\left(\phi_{i}, x, y\right)}{2 f_{3}^{2}(t, x, y, \Theta)}\right)\right),
\end{gathered}
$$

where, 
$f_{5}\left(\phi_{i}, x, y\right)=\frac{\left(1+f_{4}^{2}(x, y)\right)+\cos \phi\left(1-f_{4}^{2}(x, y)\right)}{2\|\vec{w}\|^{2}}$.

Multiplying the spillage efficiency, $f_{s p}(t, x, y, \Theta)$, by the solar radiation at a specific time point and by other losses inherent in the system gives us the total energy that reaches the receiver at time $t$. This value is for one heliostat, aiming at a specific aimpoint.

\section{Optimisation}

In this section our goal is to optimise the selection of aiming points and grouping strategies for the heliostats in a fixed field, in order to maximise the energy produced under some homogeneity constraints on radiation. In the first instance, we will consider a fixed field of heliostats and optimise the aiming strategy at multiple time instants across a day, in order to incorporate the effect of shadowing and blocking of the heliostats. These constraints have been incorporated using a Binary Integer Linear Programming (BILP) technique, so that the optimal grouping strategy can be found to maximise the energy received.

There are various optimisation techniques that could be applied to solve this problem, including heuristic methods such as Ant Colony or Genetic Algorithm techniques, however the BILP technique was implemented in this case. BILP techniques lead to the optimal solution within a finite time period, whereas this is not guaranteed with purely heuristic methods and is therefore not the best choice for this problem. Applying the BILP technique with a heuristic time limit allows a solution to be obtained quickly, whilst the gap between the obtained solution and the optimal solution will be between a defined upper bound.

Changing the aim point of a heliostat from the centre of the receiver to another point affects the amount of energy reaching the receiver by changing the slant range (that is, the modulus of $\vec{w}$ ), the spillage efficiency, the cosine efficiency and the light distribution across the surface.

Let $A$ be the set of aiming points on the receiver surface and let $H$ be the set of heliostats aiming at the points in $A$. Let us define an optimisation procedure for any fixed time instant, $t$.

For $h \in H, a \in A$, set $z_{h a}$ to the boolean variable defined as:

$z_{h a}= \begin{cases}1, & \text { if heliostat } h \text { is allocated to aiming point } a \\ 0, & \text { otherwise }\end{cases}$

The reflected radiation pattern $r_{h a}^{b}$ is the radiation point value at aiming point $b$ received from heliostat $h$ aiming at aiming point $a$. The total integrated radiation, $R_{h a}$, is the total radiation received across the receiver from heliostat $h$ aiming at aiming point $a$.

We therefore look to maximise the total incident energy on the receiver:

Maximise $\sum_{a, h} R_{h a} z_{h a}$.

We constrain this objective function by requiring that no heliostat may be looking at more than one aiming point on the receiver, but may be stowed in case of high winds or potential damage to the receiver or mirror.

This gives the constraint:

$\sum_{h} z_{h a} \leq 1 \quad \forall a \in A$

We also constrain the received energy at the aiming points:
$C_{*} \leq \sum_{h, a} r_{h a}^{b} z_{h a} \leq C^{*} \quad \forall b \in A$,

where $C^{*}$ is a fixed maximum energy and $C_{*}$ is a fixed minimum energy. These constraints prevent the receiver being subject to excessive temperatures (which could cause permanent damage) and also ensure that a minimum amount of energy is being collected at each aiming point.

In order to approximate a uniform distribution of energy across the receiver, we will look to also constrain the range of energy received between any two aiming points by imposing

$\max _{a} \sum_{h, a} r_{h a}^{i} z_{h a}-\min _{a} \sum_{h, a} r_{h a}^{j} z_{h a} \leq \tau$

where $\tau$ is a given constant.

This can also be written in the form

$\max _{a} \sum_{h, a} r_{h a}^{i} z_{h a} \leq \tau+\min _{a} \sum_{h, a} r_{h a}^{j} z_{h a}$

which is equivalent to the following set of linear constraints:

$\sum_{h, a} r_{h a}^{i} z_{h a} \leq \tau+\sum_{h, a} r_{h a}^{j} z_{h a} \quad \forall i, j \in A$, with $i \neq j$

The optimisation problem to be solved at each time instant $t$ can then be summarised as follows:

Maximise $\sum_{\mathrm{h}, \mathrm{a}} R_{h a} z_{h a}$

Subject to:

$\sum_{h} z_{h a} \leq 1 \quad \forall a \in A$,

$C_{*} \leq \sum_{h \in H} r_{h a}^{i} z_{h a} \leq C^{*} \quad \forall i \in A$,

$\sum_{\substack{h \in H \\ a \in A}} r_{h a}^{i} z_{h a} \leq \tau+\sum_{\substack{h \in H \\ a \in A}} r_{h a}^{j} z_{h a} \quad \forall i, j \in A$ with $i \neq j$

$z_{\text {ha }} \in\{0,1\} \quad \forall h \in H, \forall a \in A$

The optimal aiming strategy for a SPT plant is dependent on the time of day, as well as the day of the year. For an optimal aiming strategy to be achieved, it must be optimised at a rate that will capture the changing radiation pattern over time.

In terms of the optimisation problem, both the objective function and the constraints will change as functions of time, caused by the variable incident radiation and physical constraints on the SPT plant. A rapid change in incident radiation at one point on the receiver surface, for instance caused by passing clouds, could potentially cause damage, indicating that frequent updates to the aiming strategy are needed.

As there is no guarantee that the optimal aiming pattern will remain optimal, or even feasible, over time, the optimisation procedure must be repeated frequently, using knowledge of local weather to constrain the problem in real-time.

The next part of this paper applies this optimisation method to a SPT plant and demonstrates its efficiency with near real-time 


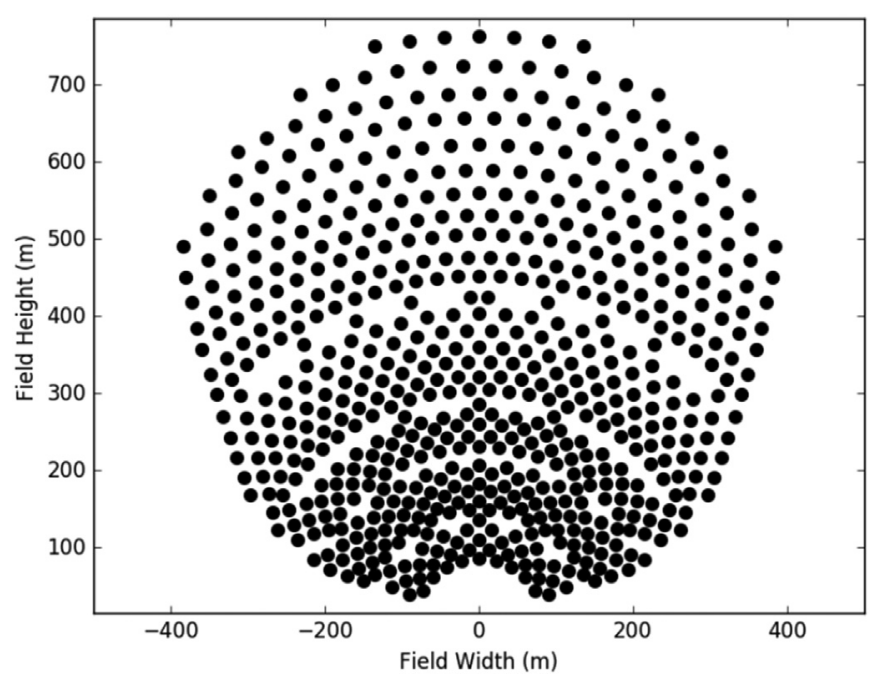

Fig. 2. PS10 layout.

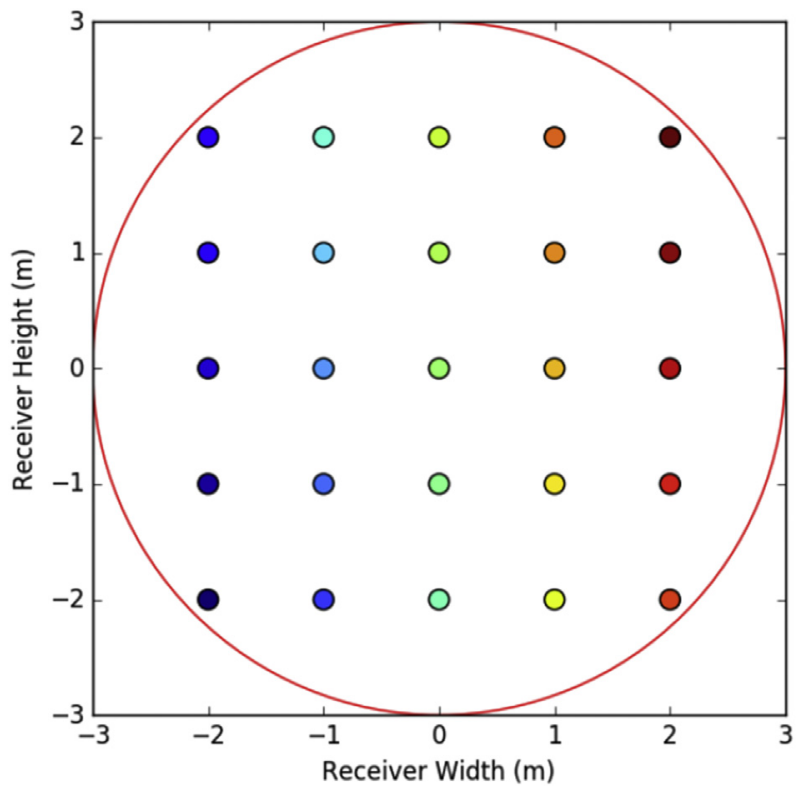

Fig. 3. Aiming points.

updates to the optimal aiming strategy during operation of the plant.

\section{Results}

For comparison against other research, we apply the aforementioned optimisation procedure with a grid of 25 aiming points to the PS10 plant in Sanlucar la Mayor, Seville [1]. Observe that in this field all heliostats are identical, but the presented approach works for fields of heliostats with different sizes, such as those considered in Ref. [13].

The aiming points allocated to the heliostats are colour coded according to the colours shown in Fig. 3 and the location of the heliostats within the SPT field are shown in Fig. 2, where the x-axis goes from East to West.

The optimisation procedure is coded in Python, using the Gurobi optimisation package [2]. The optimisation problems to be solved are difficult in short time scales, due to the large number of boolean

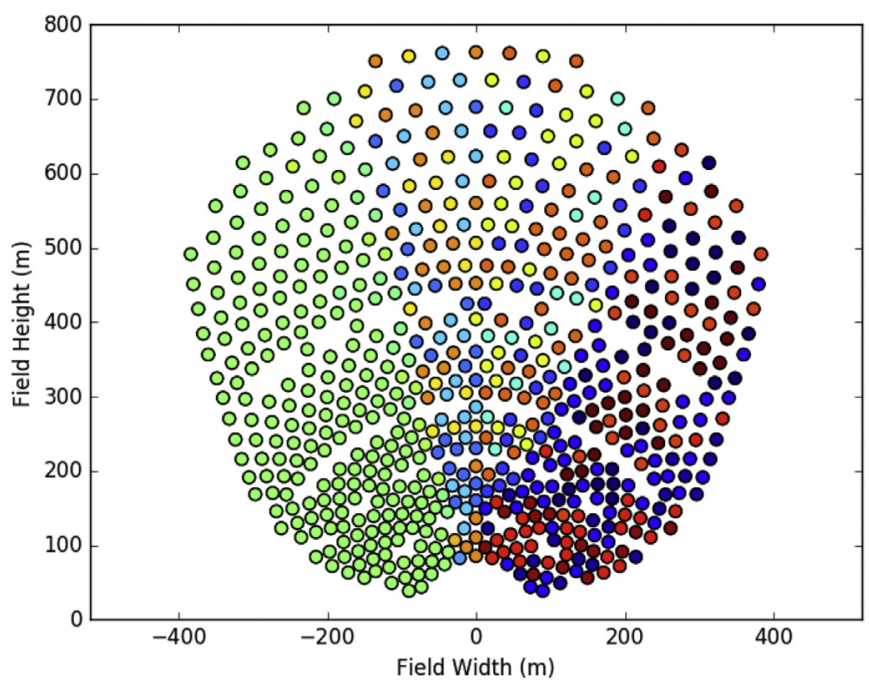

Fig. 4. 6am allocation.

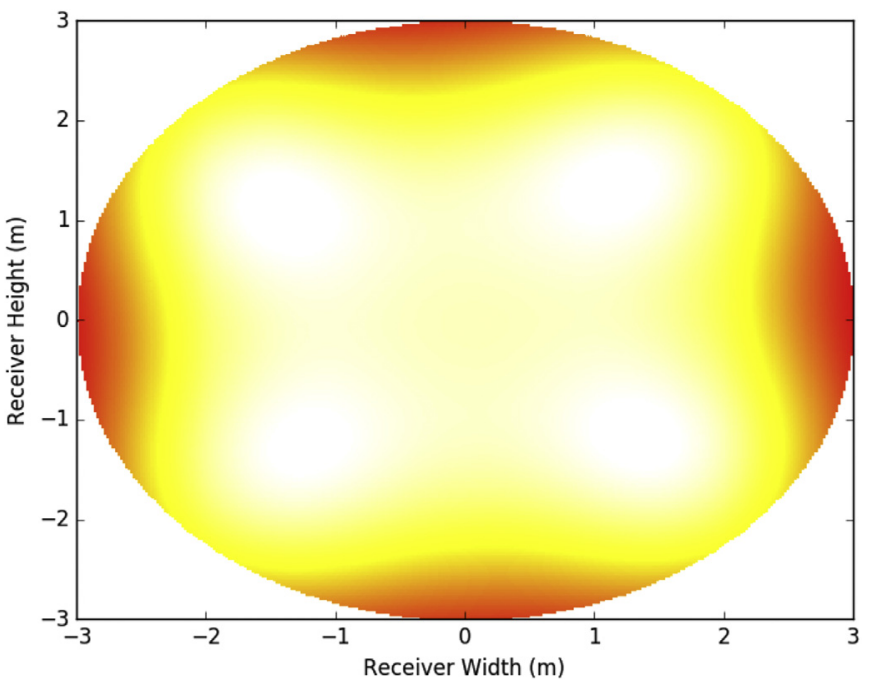

Fig. 5. 6am distribution.

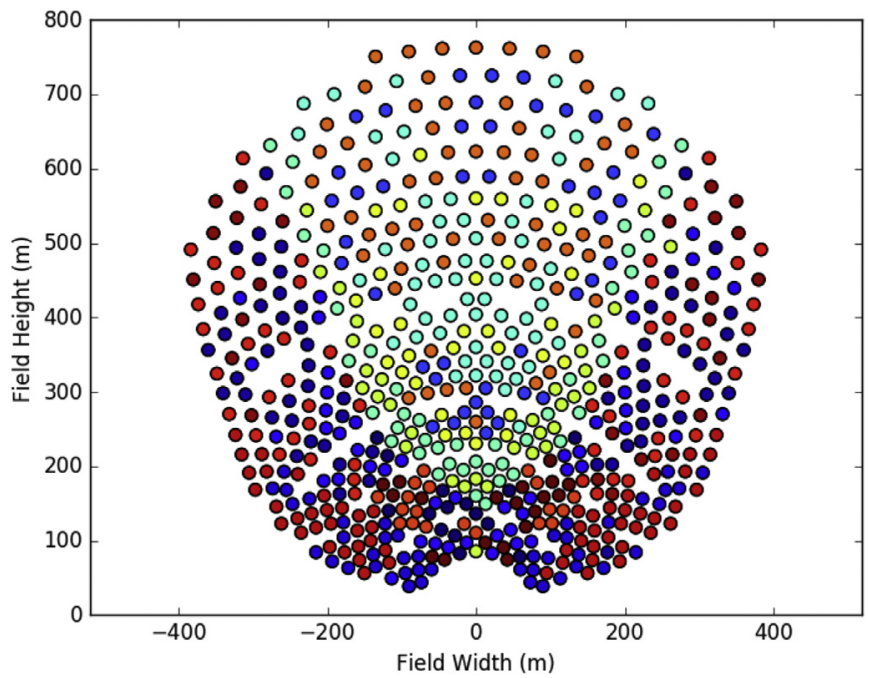

Fig. 6. $12 \mathrm{pm}$ allocation. 


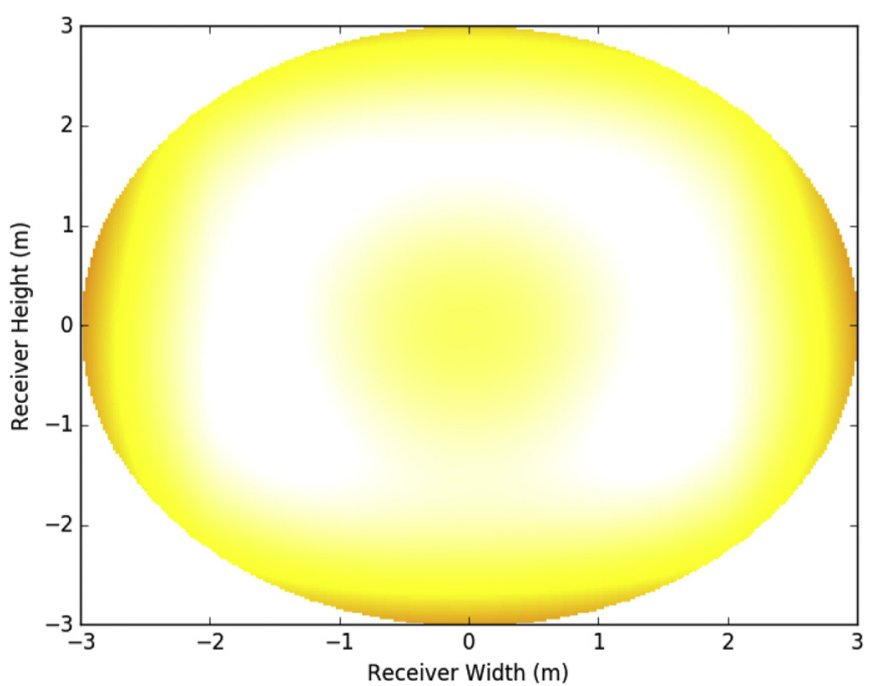

Fig. 7. $12 \mathrm{pm}$ distribution.

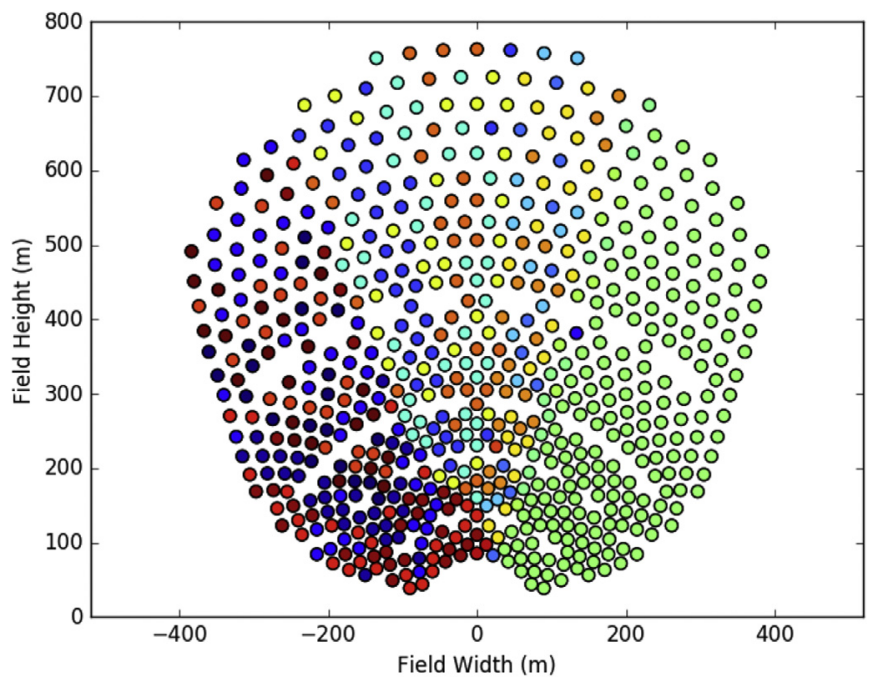

Fig. 8. 6pm allocation.

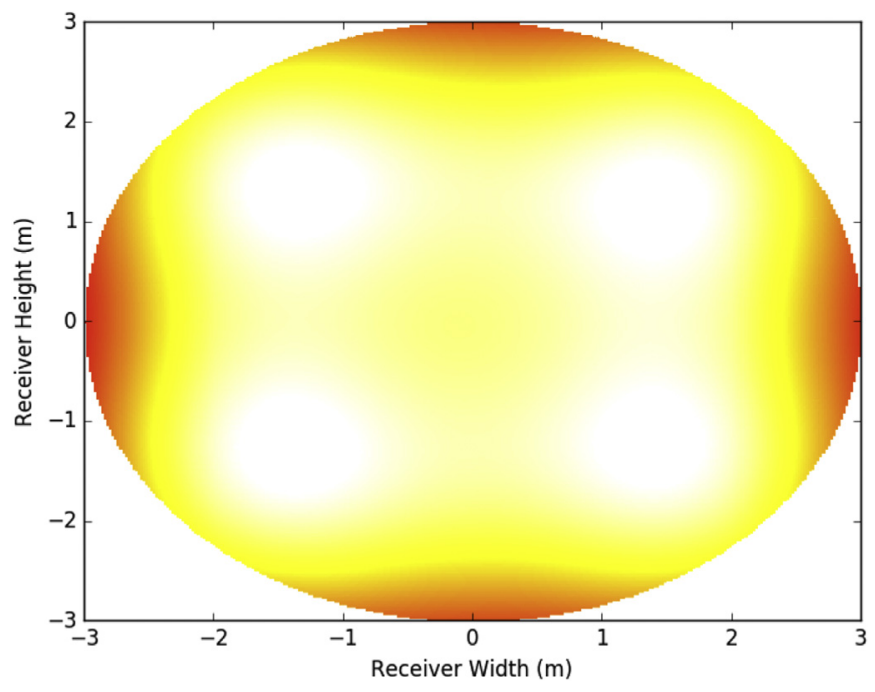

Fig. 9. 6pm distribution. variables. However, running an integer programming solver with a short time limit of $30 \mathrm{~s}$ was found to approximate the optimal solution closely and leads to near real-time satisfactory updates to the aiming strategy.

The values for the maximum, minimum and range constraints used in the analysis for this paper were calculated in order to reflect working values that an SPT plant would define, based upon physical limits of the components. For this purpose, the problem is solved to optimality, without considering any constraints, to find the maximum energy at over the aiming points. The problem is then constrained to $25 \%$ of this maximum and optimised in 30 s, with the resulting range then constrained to $15 \%$ of its value and used to find the constrained result.

The results in Figs. 4-9 show the computed optimal aiming strategy and energy distribution, for three different times across a day. During the first solar hour of the day, shown in Fig. 4, the heliostats located on the West side of this Northern hemisphere plant have the smallest cosine angle, and are therefore aiming at the receiver edges. The heliostats on the East side have a larger cosine angle, and are therefore aimed at the centre of the receiver.

This result concurs with the distribution found in (1), where having a smaller cosine efficiency causes the distribution of energy on the receiver surface to be larger. The heliostats aiming at the centre have the worst efficiency, and are aiming there to not lose as much energy. The heliostats with better efficiencies are therefore aiming elsewhere, as they don't lose as much energy by looking towards the edges.

To demonstrate the use of near real-time updates, we can look at the evolution of the aiming strategy over smaller timesteps. This will utilise SPT plant operators knowledge of local weather conditions and predictive technology to recalculate the optimal solution and react to changing weather conditions.

Passing clouds over a large heliostat field cause groups of heliostats to become less efficient, which will therefore change the optimal aiming strategy. The effect of clouds on CSP technology is of importance in the efficiency of a SPT plant, and has prompted the development of technology to predict the quantity and location of clouds, see Refs. $[3,4,19]$. Standard procedure in order to prevent thermal shock to the receiver when a cloud passes by, is to aim heliostats away [19], which will reduce overall energy collected.

Using the location and size of a cloud in the optimisation procedure outlined in this paper allows an SPT plant to further optimise aiming strategies in near real-time, by re-optimising the

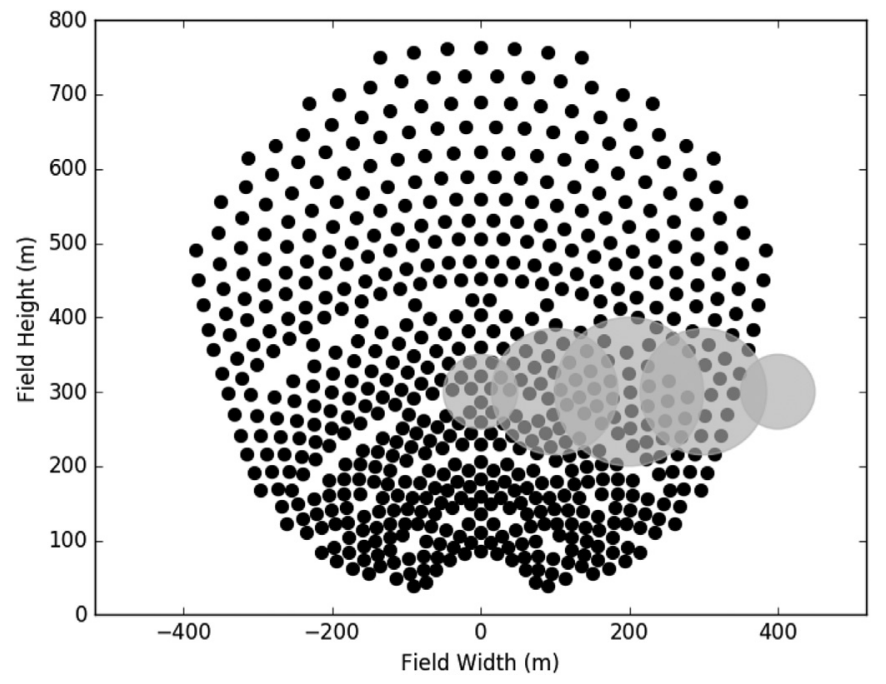

Fig. 10. Cloud example. 


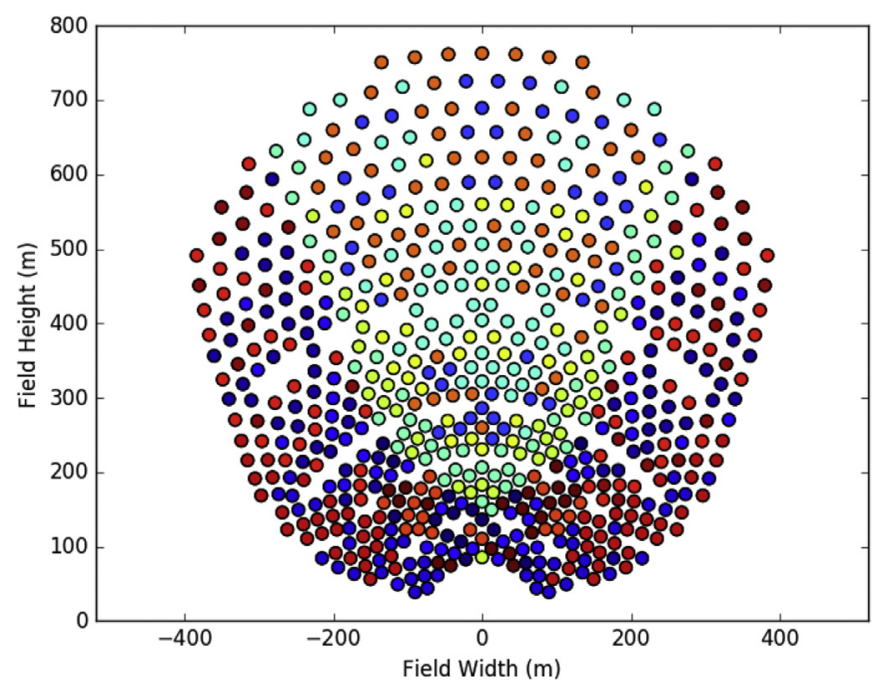

Fig. 11. 12pm allocation.

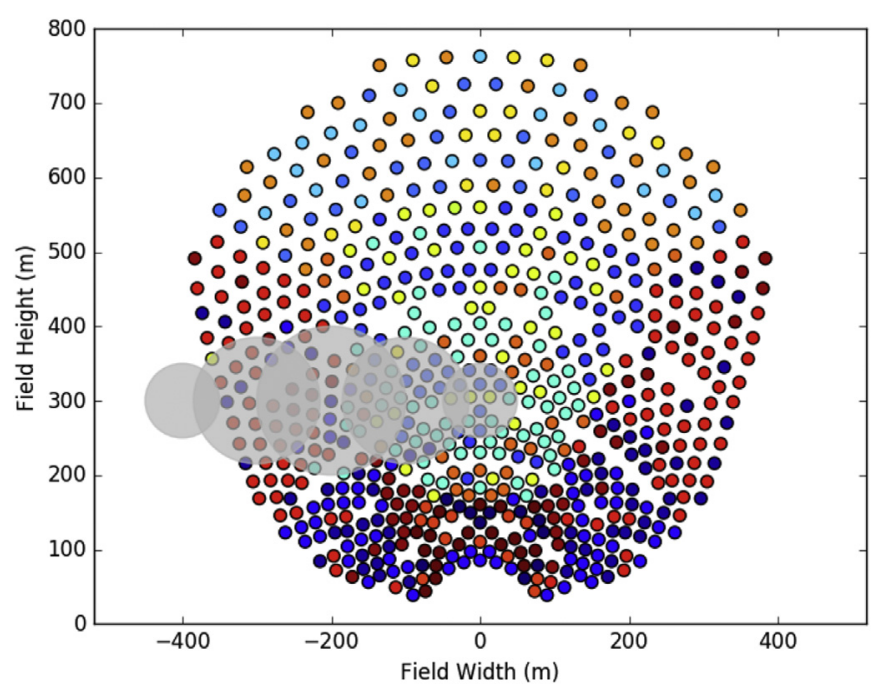

Fig. 12. 12pm distribution with cloud.

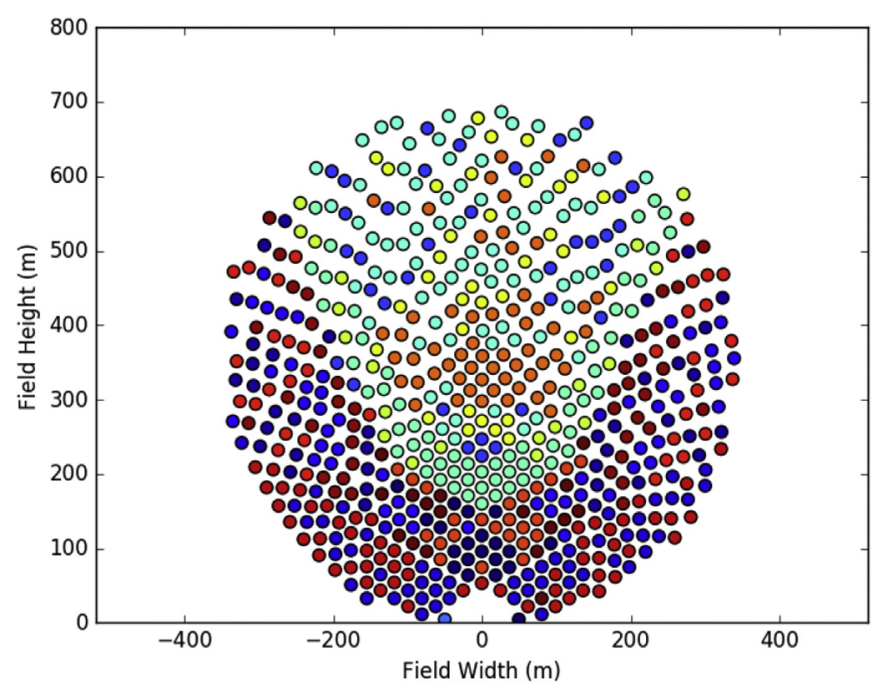

Fig. 13. 12pm allocation (Greedy field).

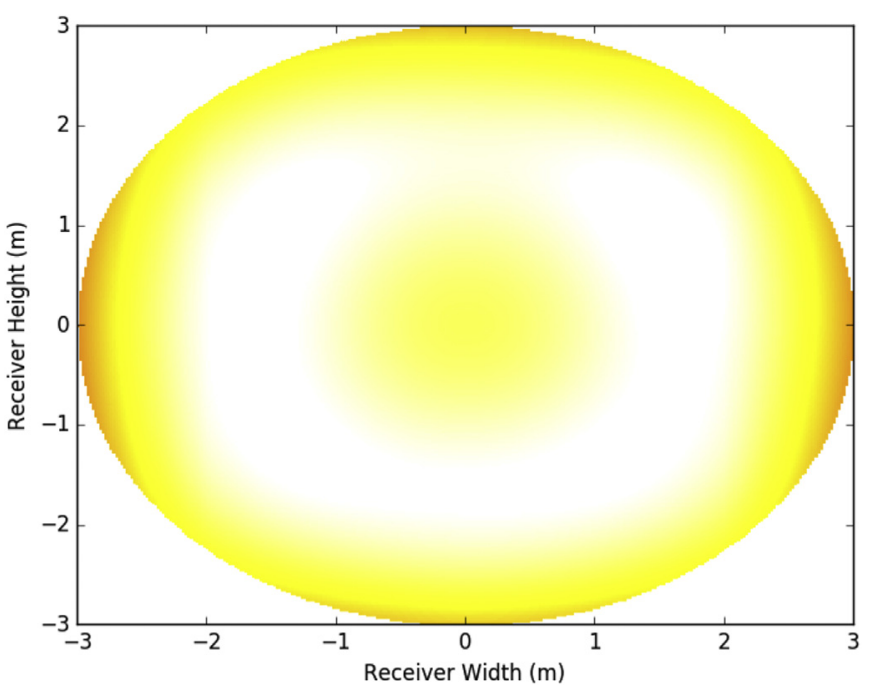

Fig. 14. 12pm distribution (Greedy field).

strategy whilst taking into account constraints such as cloud passage. The size, shape, and location of a cloud may be changed within the code, where the heliostats that are covered are assumed to suffer a $70 \%$ drop in efficiency. An example of cloud implementation is furnished in Fig. 10. Figs. 11 and 12 show a comparison of the optimal results for $12 \mathrm{pm}$, with and without a cloud, where it can be seen that the presence of a cloud alters the optimal aiming strategy.

Our approach consists of solving an Integer Linear Programming problem, which makes no assumption on the heliostat field layout. Therefore, it is also applicable to irregularly distributed heliostats, such as those generated with the Greedy Algorithm developed in Ref. [11]. This is demonstrated in Figs. 13 and 14 for the irregular field in Ref. [11].

\section{Conclusions}

An aiming strategy has been developed to optimise the energy collected by an SPT plant at a near real-time scale, considering multiple constraints based upon physical requirements. This procedure has been demonstrated at multiple time points across a day and considered cloud effects and changing the shape of the heliostat field, showing that the procedure may be applied to any plant design and can be used to react to inclement weather whilst maintaining optimal energy production.

A linear integer problem with a short time limit was solved using an integer programming solver, without the use of additional preprocessing. It is expected that better results may be obtained if the solver is given a reasonably good starting solution, obtained by an ad hoc procedure which exploits the problem structure.

The energy distribution and aiming strategies presented are a theoretical representation of SPT performance, and should be validated against practical results from experiments with a SPT plant. This validation should be completed with the cooperation of a SPT plant operator and investigate the effects of implementing different aiming strategies and compare them with theoretical results.

The effect of inclement weather on the optimal aiming strategy employed has been demonstrated here to show capability of the model developed, and further research should be conducted to investigate what different types of inclement weather do to the aiming strategy. 
Changing the location and quantity of aiming points on the receiver surface will affect the optimal solution. Increasing the number of aiming points may lead to a solution which provides a smoother distribution and higher energy generation, but it will increase the computational time considerably. Further research into the optimisation of aiming strategy and aiming point locations at the same time should be considered, where a variety of optimisation techniques could be compared.

An interesting extension of the present work is the case in which there are multiple receivers [12,24], where the aiming strategy must be optimised using aiming points distributed across multiple locations. If the plant design is already given, the model considered in this paper extends in a straightforward manner to this problem. If, on the contrary, the plant is to be designed, an alternating approach can be considered to optimise both the aiming strategy and the heliostat locations within the field.

\section{Acknowledgements}

This research is being supported by the Spanish Government through the research projects PCIN-2015-108, P11-FQM-7603, MTM2015-65915-R and MTM2016-76990-P at the University of Seville.

\section{References}

[1] Abengoa PS10 SPT Plant. http://www.abengoasolar.com/web/en/plantas solares-/plantas_para_terceros/espana/index.html [Accessed 22 February 2017].

[2] Gurobi Optimization Solver. http://www.gurobi.com/[Accessed 22 February 2017].

[3] Alonso J, Batlles FJ, López G, Ternero A. Sky camera imagery processing based on a sky classification using radiometric data. Energy 2014:68(May):599-608.

[4] Alonso-Montesinos J, Batlles FJ. Solar radiation forecasting in the short- and medium-term under all sky conditions. Energy 2015;83:387-93.

[5] Astolfi Marco, Binotti Marco, Mazzola Simone, Zanellato Luca, Manzolini Giampaolo. Heliostat aiming point optimization for external tower receiver. Sol Energy 2016:1-16.

[6] Baños R, Manzano-Agugliaro F, Montoya FG, Gil C, Alcayde A, Gómez J. Optimization methods applied to renewable and sustainable energy: a review. Renew Sustain Energy Rev 2011;15(4):1753-66.

[7] Barberena JG, Mutuberria Larrayoz A, Sánchez M, Bernardos A. State-of-theart of heliostat field layout algorithms and their comparison. Energy Procedia 2016:93(March):31-8.

[8] Belhomme Boris, Pitz-Paal Robert, Schwarzbözl Peter. Optimization of heliostat aim point selection for central receiver systems based on the Ant Colony optimization metaheuristic. J Sol Energy Eng 2013;136(February 2014). 011005.
[9] Berenguel M, Rubio FR, Valverde A, Lara PJ, Arahal MR, Camacho EF, et al. An artificial vision-based control system for automatic heliostat positioning offset correction in a central receiver solar power plant. Sol Energy 2004;76(5): $563-75$.

[10] Besarati Saeb M, Yogi Goswami D. A computationally efficient method for the design of the heliostat field for solar power tower plant. Renew Energy 2014;69(November 2016):226-32.

[11] Carrizosa E, Domínguez-Bravo C, Fernández-Cara E, Quero M. A heuristic method for simultaneous tower and pattern-free field optimization on solar power systems. Comput Oper Res 2015;57:109-22.

[12] Carrizosa E, Domínguez-Bravo C, Fernández-Cara E, Quero M. Optimization of multiple receivers solar power tower systems. Energy 2015;90:2085-93.

[13] Carrizosa E, Domínguez-Bravo Carmen-Ana, Fernández-Cara E, Quero M. An optimization tool to design the field of a solar power tower plant allowing heliostats of different sizes. Int J Energy Res 2017;41(8):1096-107.

[14] Collado FJ, Gómez A, Turégano JA. An analytic function for the flux density due to sunlight reflected from a heliostat. Sol Energy 1986;37(3):215-34.

[15] Collado Francisco J. Quick evaluation of the annual heliostat field efficiency. Sol Energy 2008;82(4):379-84

[16] Cruz NC, Redondo JL, Álvarez JD, Berenguel M, Ortigosa PM. A parallel Teaching-Learning-Based Optimization procedure for automatic heliostat aiming. J Supercomput 2016;73(1):591-606.

[17] Fend Thomas, Hoffschmidt Bernhard, Pitz-Paal Robert, Reutter Oliver Rietbrock Peter. Porous materials as open volumetric solar receivers: experimental determination of thermophysical and heat transfer properties. Energy 2004;29(5-6):823-33.

[18] Kribus Abraham, Vishnevetsky Irina, Yogev Amnon, Rubinov Tatiana. Closed loop control of heliostats. Energy 2004;29(5-6):905-13.

[19] Lopez-Martinez M, Rubio FR. Cloud detection system for a solar power tower plant. In: IEEE 2002 28th annual conference of the industrial electronics society. IECON 02, 3; 2002. p. 2560-5.

[20] Relloso S, García E. Tower technology cost reduction approach after gemasolar experience. Energy Procedia 2015;69:1660-6.

[21] Salomé Adrien, Chhel Fabien, Flamant Gilles, Ferrière Alain, Thiery Frederik. Control of the flux distribution on a solar tower receiver using an optimized aiming point strategy: application to THEMIS solar tower. Sol Energy 2013;94: 352-66.

[22] Sánchez-González Alberto, Reyes Rodríguez-Sánchez María, Santana Domingo. Aiming strategy model based on allowable flux densities for molten salt central receivers. Sol Energy 2015. http://dx.doi.org/10.1016/ j.solener.2015.12.055.

[23] Sánchez-González Alberto, Santana Domingo. Solar flux distribution on central receivers: a projection method from analytic function. Renew Energy 2015;74:576-87.

[24] Schmitz Mark, Schwarzbözl Peter, Buck Reiner, Pitz-Paal Robert. Assessment of the potential improvement due to multiple apertures in central receiver systems with secondary concentrators. Sol Energy 2006;80(1):111-20.

[25] Wang Kun, Ling He Ya, Qiu Yu, Zhang Yuwen. A novel integrated simulation approach couples MCRT and Gebhart methods to simulate solar radiation transfer in a solar power tower system with a cavity receiver. Renew Energy 2016;89:93-107.

[26] Yu Qiang, Wang Zhifeng, Xu Ershu. Analysis and improvement of solar flux distribution inside a cavity receiver based on multi-focal points of heliostat field. Appl Energy 2014;136:417-30. 